Punjab University Journal of Mathematics

(ISSN 1016-2526)

Vol. 53(1)(2020) pp. 19-33

\title{
$(m, n)$-Convexity-cum-Concavity on Fuzzy Soft Set with Applications in First and Second Sense
}

\author{
Atiqe Ur Rahman ${ }^{1}$, Muhammad Saeed ${ }^{2, *}$, Muhammad Arshad ${ }^{3}$, Muhammad Ihsan ${ }^{4}$, \\ Muhammad Rayees Ahmad ${ }^{5}$ \\ $1,2,3,4,5$ Department of Mathematics, \\ University of Management and Technology, Lahore, Pakistan, \\ Email: aurkhb@gmail.com ${ }^{1}$, muhammad.saeed@umt.edu.pk ${ }^{2, *}$,makkhb84@gmail.com \\ 3 , mihkhb@gmail.com ${ }^{4}$, rayeesmalik.ravian@gmail.com ${ }^{5}$
}

Received: 02 September,2020 / Accepted: 07 January, 2021 / Published online: 08 January, 2021

\begin{abstract}
Soft set theory is considered as one of the best effective tool which provides parameterization approach to tackle the inadequacy of fuzzy set. So far, it has been applied to different mathematical concepts such as set operations, algebraic structure (e.g., group and ring theory) and topological spaces. Many researchers have studied classical concept of convex and concave set under fuzzy-like, soft-like and fuzzy soft-like environments. In this paper, new notions of $(m, n)$-convex and $(m, n)$ concave fuzzy soft sets are developed first and then their versions for first and second senses are established. Further some known classical results and properties are generalized under fuzzy soft set environment. Moreover, special cases of $(m, n)$-convexity on fuzzy soft sets are established.
\end{abstract}

AMS (MOS) Subject Classification Codes: 03B52; 03E72; 52A01

Key Words: Soft Set, Convex Soft Set, Concave Soft Set, Convex Fuzzy Soft Set, Concave Fuzzy Soft Set, $(m, n)$-convex Fuzzy Soft Set, $(m, n)$-concave Fuzzy Soft Set.

\section{INTRODUCTION}

In 1965, Zadeh [34] conceptualized the theory of fuzzy sets. The theories like theory of probability, theory of fuzzy sets, and the interval mathematics, are considered as mathematical means to tackle many intricate problems involving various uncertainties, in different fields of mathematical sciences. These theories have their own complexities which restrain them to solve these problems successfully. The reason for these hurdles is, possibly, the inadequacy of the parameterization tool. A mathematical tool is needed for dealing with uncertainties which should be free of all such impediments.

In 1999, Molodtsov [21] introduced such mathematical tool called soft sets in literature as a new parameterized family of subsets of the universe of discourse. Later, Maji et al. [19], 
[20] extended the concept and introduced some fundamental terminologies and operations like equality of two soft sets, subset and super set of a soft set, complement of a soft set, null soft set, absolute soft set, AND, OR and also the operations of union and intersection. They also verified De Morgan's laws and a number of other results. They also defined fuzzy soft set and successfully applied it in decision making. Many researchers [24]-[9] extended the concept and successfully applied it in decision making and other different branches of mathematical sciences. Chaudhuri [10] studied concave fuzzy sets (converse of Zadeh's convex fuzzy set) and discussed its different properties. Many authors [14]-[25] applied this concept to other fuzzy-like environments and discussed their properties.

Deli [11] developed the concept of convexity under soft sets environment and discussed some of its properties and aggregation operations. Later, he [12] extended his concept to fuzzy soft set environment with more generalized properties. Rahman et al. [26] extended the concept to more generalized form of soft set i.e. hypersoft set and presented its important results and properties.

Convexity has an essential function in optimization and control, pattern classification and recognition, image processing and many other relating topics in different fields of mathematical sciences like operation research, numerical analysis etc. The fuzzy soft set is that effective hybrid structure which not only minimizes the complexities of fuzzy set for dealing uncertainties but also fulfills all the parameterization requirements of soft sets. This feature makes it a completely new mathematical tool for solving problems dealing with uncertainties.

Having motivation from the work of [34], [10], [11] and [12], $(m, n)$-convexity and $(m, n)$ concavity are conceptualized along with some of its generalized results and properties. Moreover, this concept is applied to different senses i.e. First and Second Senses.

The rest of the paper is organized as:

Section 2 recalls some basic terminologies and definitions from literature. Section 3 presents the basic notions of $(m, n)$-convexity and $(m, n)$-concavity on fuzzy soft sets with their properties. Section 4 discusses the application of this concept to first and second senses and then section 5 concludes the paper.

\section{PRELIMINARIES}

Here some fundamental terms and results of soft set and fuzzy soft set are presented.

Definition 1. [34] Let $\breve{Z}$ be the universe. Then, a fuzzy set $\breve{G}$ over $\breve{Z}$ is defined by a set of ordered pair $\breve{G}=\left\{\left(\zeta_{\breve{G}}(\omega) / \omega\right): \omega \in \breve{Z}\right\}$ where $\zeta_{\breve{G}}: \breve{Z} \rightarrow[0,1]$ is called membership function of $G$. The value $\zeta_{\breve{G}}(\omega)$ is called the membership value or the grade of membership of $\omega \in \breve{Z}$. The membership value represents the degree of $\omega$ belonging to the fuzzy set $\breve{G}$.

Definition 2. [10] A fuzzy set $\breve{G}$ in $R^{n}$ is said to be convex if

$$
\zeta(\gamma) \geq \min \{\zeta(\alpha), \zeta(\beta)\}
$$

where $\alpha, \beta \in R^{n}$ and $\gamma$ on the line segment $\overline{\alpha \beta}$. Similarly it is called concave if

$$
\zeta(\gamma) \leq \min \{\zeta(\alpha), \zeta(\beta)\}
$$

Definition 3. [21] Let $\breve{P}(\breve{U})$ be the power set of $\breve{U}$ (universe of discourse) and $\breve{L}$ be a set of parameters defining $\breve{U}$. A soft set $\breve{M}$ over $\breve{U}$ is a set defined by a set valued function $\breve{M}$ 
representing a mapping

$$
\breve{h}_{\breve{M}}: \breve{L} \rightarrow P(\breve{U})
$$

Definition 4. [19] If $\breve{h}_{\breve{M}}(\omega) \subseteq \breve{h}_{\breve{N}}(\omega)$ for all $\omega \in \breve{L}$, then $\breve{M}$ is a soft subset of $\breve{N}$, denoted by $\breve{M} \widetilde{\subseteq} \breve{N}$

Definition 5. [19] Union of sets $\breve{M}$ and $\breve{N}(M \widetilde{U} N)$ is defined as

$$
\breve{h}_{\breve{M} \widetilde{U} \breve{N}}(\omega)=\breve{h}_{\breve{M}}(\omega) \cup \breve{h}_{\breve{N}}(\omega) \forall \omega \in \breve{L}
$$

Definition 6. [19] Intersection of set $\breve{M}$ and $\breve{N}(\breve{M} \widetilde{\cap} \breve{N})$ is defined as

$$
\breve{h}_{\breve{M} \widetilde{\cap} \breve{N}}(\omega)=\breve{h}_{\breve{M}}(\omega) \cap \breve{h}_{\breve{N}}(\omega) \forall \omega \in \breve{L}
$$

Definition 7. [11] The $\breve{\delta}-$ inclusion of a soft set $\breve{M}$ (where $\breve{\delta} \tilde{\subseteq} \breve{U}$ ) is defined by

$$
\breve{M}^{\breve{\delta}}=\{\omega \in \breve{L}: \breve{h} \breve{M}(\omega) \supseteq \breve{\delta}\}
$$

Definition 8. [11]

The soft set $\breve{M}$ on $\breve{L}$ is called a convex soft set if

$$
\breve{h}_{\breve{M}}(\epsilon \omega+(1-\epsilon) \mu) \supseteq \breve{h}_{\breve{M}}(\omega) \cap \breve{h}_{\breve{M}}(\mu)
$$

for every $\omega, \mu \in \breve{L}$ and $\epsilon \in \breve{J}$.

Definition 9. [11]

The soft set $\breve{M}$ on $\breve{L}$ is called a concave soft set if

$$
\breve{h}_{\breve{M}}(\epsilon \omega+(1-\epsilon) \mu) \subseteq \breve{h}_{\breve{M}}(\omega) \cup \breve{h}_{\breve{M}}(\mu)
$$

for every $\omega, \mu \in \breve{L}$ and $\epsilon \in \breve{J}$.

Definition 10. [20] Let $\breve{Z}$ be an initial universe, $\breve{G}(\breve{Z})$ be all fuzzy sets over $\breve{Z}$. $\breve{H}$ be the set of all parameters and $\breve{J} \subseteq \breve{H}$. An fuzzy soft set $\Gamma_{\breve{J}}$ on the universe $\breve{Z}$ is defined by the set of ordered pairs as follows,

$$
\Gamma_{\breve{J}}=\left\{\left(\omega, \gamma_{\breve{J}}(\omega)\right): \omega \in \breve{H}, \gamma_{\breve{J}}(\omega) \in \breve{G}(\breve{Z})\right\}
$$

where $\gamma_{\breve{J}}(\omega): \breve{H} \rightarrow \breve{G}(\breve{Z})$ such that $\gamma_{\breve{J}}(\omega)=\emptyset$ if $\omega \notin \breve{J}$, and for all $\omega \in \breve{H}$

$$
\Gamma_{\breve{J}}=\left\{\mu_{\Gamma_{\breve{J}}}(\sigma) / \sigma: \sigma \in \breve{Z}, \mu_{\Gamma_{\breve{J}}}(\sigma) \in[0,1]\right\}
$$

is a fuzzy set over $Z$.

Definition 11. [12]

The fuzzy soft set $\Gamma_{\breve{M}}$ on $\breve{L}$ is called a convex Fuzzy soft set if

$$
\breve{\gamma}_{\breve{M}}(\epsilon \omega+(1-\epsilon) \mu) \supseteq \breve{\gamma}_{\breve{M}}(\omega) \cap \breve{\gamma}_{\breve{M}}(\mu)
$$

for every $\omega, \mu \in \breve{L}$ and $\epsilon \in \breve{J}$.

Definition 12. [12]

The fuzzy soft set $\Gamma_{\breve{M}}$ on $\breve{L}$ is called a concave Fuzzy soft set if

$$
\breve{\gamma}_{\breve{M}}(\epsilon \omega+(1-\epsilon) \mu) \subseteq \breve{\gamma}_{\breve{M}}(\omega) \cup \breve{\gamma}_{\breve{M}}(\mu)
$$

for every $\omega, \mu \in \breve{L}$ and $\epsilon \in \breve{J}$. 


\section{3. $(m, n)$-CONVEX AND $(m, n)$-CONCAVE FuZZY SOFT SETS}

In this section, $(m, n)$-Convex and $(m, n)$-Concave Fuzzy soft sets are defined and then some desired results are proved. In this paper, $\breve{L}$ will play the role of $\mathbb{R}^{n}$ and $\breve{U}$ denotes the arbitrary set.

Definition 13. The fuzzy soft set $\Gamma_{\breve{A}}$ on $\breve{L}$ is called $(m, n)$-convex FSS if

$$
\gamma_{\breve{A}}\left(n \omega_{1}+m(1-n) \omega_{2}\right) \supseteq \gamma_{\breve{A}}\left(\omega_{1}\right) \cap \gamma_{\breve{A}}\left(\omega_{2}\right)
$$

for $\omega_{1}, \omega_{2} \in \breve{L}, m \in \breve{J}$ and $n \in(0,1]$.

Example 3.1. Consider a set of mobiles as a universe of discourse $\breve{U}=\left\{M_{1}, M_{2}, M_{3}, \ldots, M_{10}\right\}$.

The attributes of mobiles under consideration form the set $\breve{A}=\left\{\omega_{1}, \omega_{2}, \omega_{3}, \omega_{4}\right\}$, where

$\omega_{1}=$ Size

$\omega_{2}=$ Camera Resolution

$\omega_{3}=\mathrm{RAM}$

$\omega_{4}=$ Colour

Now the fuzzy soft set $\left(\gamma_{\breve{A}}, \breve{A}\right)$ is a function defined by the mapping $\gamma_{\breve{A}}: \breve{A} \rightarrow \breve{F}(\breve{U})$ where $\breve{F}(\breve{U})$ is a collection of all fuzzy subsets over $\breve{U}$. Consider

$$
\begin{gathered}
\gamma_{\breve{A}}\left(\omega_{1}\right)=\left\{0.01 / M_{1}, 0.05 / M_{5}\right\} \\
\gamma_{\breve{A}}\left(\omega_{2}\right)=\left\{0.01 / M_{1}, 0.03 / M_{3}, 0.04 / M_{4}\right\} \\
\gamma_{\breve{A}}\left(\omega_{3}\right)=\left\{0.02 / M_{2}, 0.03 / M_{3}, 0.06 / M_{6}\right\} \\
\gamma_{\breve{A}}\left(\omega_{3}\right)=\left\{0.03 / M_{3}, 0.07 / M_{7}, 0.09 / M_{9}\right\}
\end{gathered}
$$

Now

$$
\begin{gathered}
\gamma_{\breve{A}}\left(\omega_{1}\right) \cap \gamma_{\breve{A}}\left(\omega_{2}\right)=\left\{0.01 / M_{1}, 0.05 / M_{5}\right\} \cap\left\{0.01 / M_{1}, 0.03 / M_{3}, 0.04 / M_{4}\right\} \\
\gamma_{\breve{A}}\left(\omega_{1}\right) \cap \gamma_{\breve{A}}\left(\omega_{2}\right)=\left\{0.01 / M_{1}\right\}
\end{gathered}
$$

If we take $n=0.1 \in(0,1]$ and $m=1.0 \in \breve{J}$, then

$$
\begin{gathered}
\gamma_{\breve{A}}\left(n \omega_{1}+m(1-n) \omega_{2}\right)=\gamma_{\breve{A}}\left(0.1 \omega_{1}+1.0(1-0.1) \omega_{2}\right) \\
\gamma_{\breve{A}}\left(n \omega_{1}+m(1-n) \omega_{2}\right)=\gamma_{\breve{A}}\left(0.1 \omega_{1}+1.0(0.9) \omega_{2}\right) \\
\gamma_{\breve{A}}\left(n \omega_{1}+m(1-n) \omega_{2}\right)=\gamma_{\breve{A}}\left(0.1 \omega_{1}+0.9 \omega_{2}\right)
\end{gathered}
$$

Consider the weight values assigned to each attribute are: $\omega_{1}=1.0, \omega_{2}=2.0, \omega_{3}=$ $3.0, \omega_{4}=4.0$, then

$$
\gamma_{\breve{A}}\left(n \omega_{1}+m(1-n) \omega_{2}\right)=\gamma_{\breve{A}}(0.1(1)+0.9(2))
$$




$$
\begin{gathered}
\gamma_{\breve{A}}\left(n \omega_{1}+m(1-n) \omega_{2}\right)=\gamma_{\breve{A}}(0.1+1.8) \\
\gamma_{\breve{A}}\left(n \omega_{1}+m(1-n) \omega_{2}\right)=\gamma_{\breve{A}}\left(1.9 \approx 2.0=\omega_{2}\right) \\
\gamma_{\breve{A}}\left(n \omega_{1}+m(1-n) \omega_{2}\right)=\left\{0.01 / M_{1}, 0.03 / M_{3}, 0.04 / M_{4}\right\}
\end{gathered}
$$

From equations ( 3.2 ) and ( 3.3 ), we have

$$
\gamma_{\breve{A}}\left(n \omega_{1}+m(1-n) \omega_{2}\right) \supseteq \gamma_{\breve{A}}\left(\omega_{1}\right) \cap \gamma_{\breve{A}}\left(\omega_{2}\right)
$$

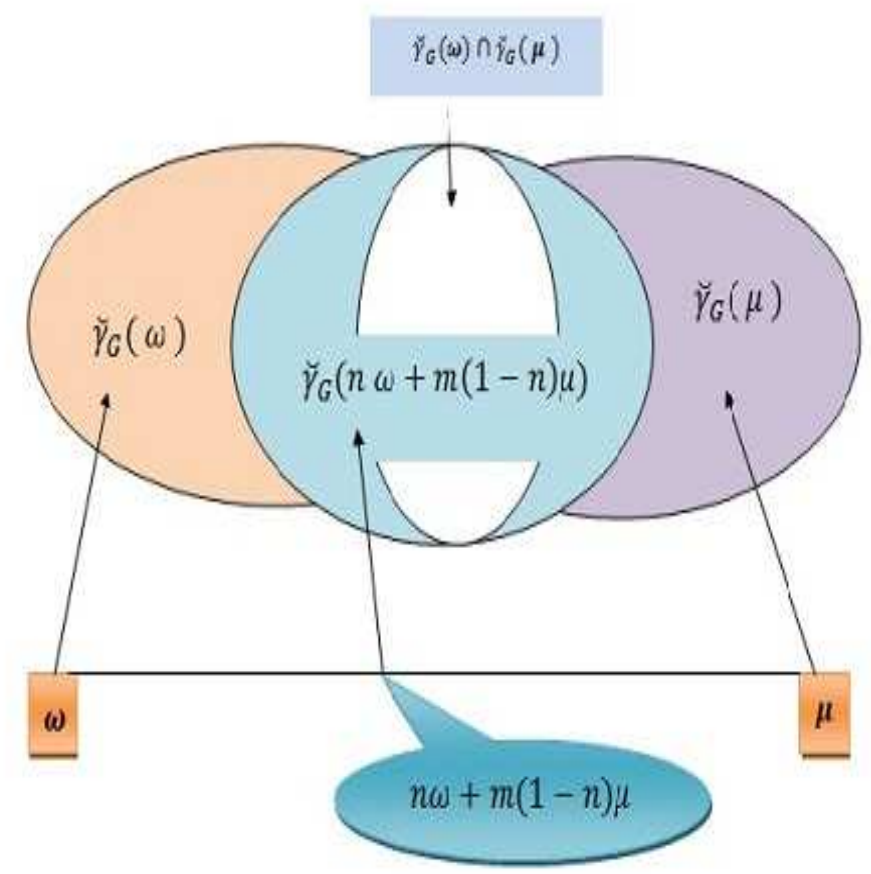

Figure 1. Convex Fuzzy soft Set

Theorem 3.2. $\Gamma_{\breve{M}} \cap \Gamma_{\breve{N}}$ is $(m, n)$-convex $F S S$ when both $\Gamma_{\breve{M}}$ and $\Gamma_{\breve{N}}$ are $(m, n)$-convex FSS.

Proof. Suppose that for $\omega_{1}, \omega_{2} \in \breve{L}$, and $\Gamma_{\breve{W}}=\Gamma_{\breve{M}} \cap \Gamma_{\breve{N}}$, Then,

$$
\gamma_{\breve{W}}\left(n \omega_{1}+m(1-n) \omega_{2}\right)=\gamma_{\breve{M}}\left(n \omega_{1}+m(1-n) \omega_{2}\right) \cap \gamma_{\breve{N}}\left(n \omega_{1}+m(1-n) \omega_{2}\right)
$$

As $\Gamma_{\breve{M}}$ and $\Gamma_{\breve{N}}$ are $(m, n)$-convex,

$$
\gamma_{\breve{M}}\left(n \omega_{1}+m(1-n) \omega_{2}\right) \supseteq \gamma_{\breve{M}}\left(\omega_{1}\right) \cap \gamma_{\breve{M}}\left(\omega_{2}\right)
$$


which implies

$$
\gamma_{\breve{N}}\left(n \omega_{1}+m(1-n) \omega_{2}\right) \supseteq \gamma_{\breve{N}}\left(\omega_{1}\right) \cap \gamma_{\breve{N}}\left(\omega_{2}\right)
$$

$$
\gamma_{\breve{W}}\left(n \omega_{1}+m(1-n) \omega_{2}\right) \supseteq\left(\gamma_{\breve{M}}\left(\omega_{1}\right) \cap \gamma_{\breve{M}}\left(\omega_{2}\right)\right) \cap\left(\gamma_{\breve{N}}\left(\omega_{1}\right) \cap \gamma_{\breve{N}}\left(\omega_{2}\right)\right)
$$
and thus

$$
\gamma_{\breve{W}}\left(n \omega_{1}+m(1-n) \omega_{2}\right) \supseteq \gamma_{\breve{W}}(\omega) \cap \gamma_{\breve{W}}\left(\omega_{2}\right)
$$

Theorem 3.3. $\Gamma_{\breve{A}}$ is $(m, n)$-convex FSS on $\breve{L}$ iff for every $n \in[0,1]$ and $\breve{\delta} \in \breve{P}(\breve{U}), \Gamma_{\breve{A}}^{\breve{\delta}}$ is $(m, n)$-convex FSS on $\breve{L}$.

Proof. Suppose $\Gamma_{\breve{A}}$ is $(m, n)$-convex FSS. If $\omega_{1}, \omega_{2} \in \breve{L}$ and $\breve{\delta} \in \breve{P}(\breve{U})$, then $\gamma_{\breve{A}}\left(\omega_{1}\right) \supseteq$ $\breve{\delta}$ and $\gamma_{\breve{A}}\left(\omega_{2}\right) \supseteq \breve{\delta}$ which means

$$
\begin{gathered}
\gamma_{\breve{A}}\left(\omega_{1}\right) \cap \gamma_{\breve{A}}\left(\omega_{2}\right) \supseteq \breve{\delta} \\
\gamma_{\breve{A}}\left(n \omega_{1}+m(1-n) \omega_{2}\right) \supseteq \gamma_{\breve{A}}\left(\omega_{1}\right) \cap \gamma_{\breve{A}}\left(\omega_{2}\right) \supseteq \breve{\delta} \\
\gamma_{\breve{A}}\left(n \omega_{1}+m(1-n) \omega_{2}\right) \supseteq \breve{\delta}
\end{gathered}
$$

and thus $\Gamma_{\breve{A}}^{\breve{\delta}}$ is $(m, n)$-convex FSS.

Conversely suppose that $\Gamma_{\breve{A}}^{\breve{\delta}}$ is $(m, n)$-convex FSS for every $n \in[0,1]$. For $\omega_{1}, \omega_{2} \in \breve{L}$, $\Gamma_{\breve{A}}^{\breve{\delta}}$ is $(m, n)$-convex with $\breve{\delta}=\gamma_{\breve{A}}\left(\omega_{1}\right) \cap \gamma_{\breve{A}}\left(\omega_{2}\right)$. Since $\gamma_{\breve{A}}\left(\omega_{1}\right) \supseteq \breve{\delta}$ and $\gamma_{\breve{A}}\left(\omega_{2}\right) \supseteq \breve{\delta}$, we have $\omega_{1} \in \Gamma_{\breve{A}}^{\breve{\delta}}$ and $\omega_{2} \in \Gamma_{\breve{s}}^{\breve{\delta}}$,

$\Rightarrow n \omega_{1}+m(1-n) \omega_{2} \in \Gamma_{\breve{A}}^{\breve{\delta}}$.

Therefore,

$$
\gamma_{\breve{A}}\left(n \omega_{1}+m(1-n) \omega_{2}\right) \supseteq \gamma_{\breve{A}}\left(\omega_{1}\right) \cap \gamma_{\breve{A}}\left(\omega_{2}\right),
$$

which proves the $(m, n)$-convexity of $\Gamma_{\breve{A}}$ on $\breve{L}$.

Definition 14. The fuzzy soft set $\Gamma_{\breve{A}}$ on $\breve{L}$ is called $(m, n)$-concave FSS if

$$
\gamma_{\breve{A}}\left(n \omega_{1}+m(1-n) \omega_{2}\right) \subseteq \gamma_{\breve{A}}\left(\omega_{1}\right) \cup \gamma_{\breve{A}}\left(\omega_{2}\right)
$$

for $\omega_{1}, \omega_{2} \in \breve{L}, m \in \breve{J}$ and $n \in(0,1]$.

Example 3.4. Consider the data given in example (3.1), we have $\gamma_{\breve{A}}\left(\omega_{1}\right) \cup \gamma_{\breve{A}}\left(\omega_{2}\right)=\left\{0.01 / M_{1}, 0.05 / M_{5}\right\} \cup\left\{0.01 / M_{1}, 0.03 / M_{3}, 0.04 / M_{4}\right\}$

$$
\gamma_{\breve{A}}\left(\omega_{1}\right) \cup \gamma_{\breve{A}}\left(\omega_{2}\right)=\left\{0.01 / M_{1}, 0.03 / M_{3}, 0.04 / M_{4}, 0.05 / M_{5}\right\}
$$

From equations ( 3.3 ) and ( 3.10), we have

$$
\gamma_{\breve{A}}\left(n \omega_{1}+m(1-n) \omega_{2}\right) \subseteq \gamma_{\breve{A}}\left(\omega_{1}\right) \cup \gamma_{\breve{A}}\left(\omega_{2}\right)
$$

Theorem 3.5. $\Gamma_{\breve{M}} \cup \Gamma_{\breve{N}}$ is $(m, n)$-concave FSS when both $\Gamma_{\breve{M}}$ and $\Gamma_{\breve{N}}$ are $(m, n)$-concave FSSs. 


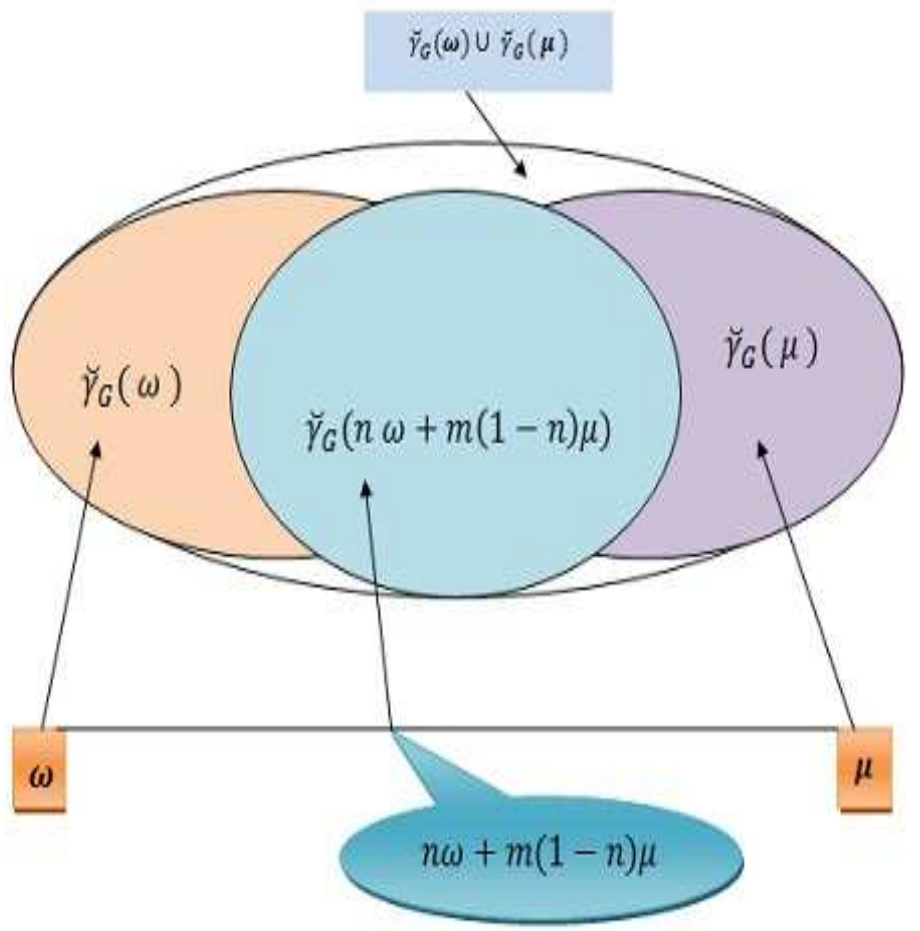

FIGURE 2. Concave Fuzzy soft Set

Proof. Suppose that for $\omega_{1}, \omega_{2} \in \breve{L}$, and $n \in \breve{J}$ and $\Gamma_{\breve{W}}=\Gamma_{\breve{M}} \cup \Gamma_{\breve{N}}$. Then,

$$
\gamma_{\breve{W}}\left(n \omega_{1}+m(1-n) \omega_{2}\right)=\gamma_{\breve{M}}\left(n \omega_{1}+m(1-n) \omega_{2}\right) \cup \gamma_{\breve{N}}\left(n \omega_{1}+m(1-n) \omega_{2}\right)
$$

Now, since $\Gamma_{\breve{M}}$ and $\Gamma_{\breve{N}}$ are $(m, n)$-concave,

$$
\begin{aligned}
& \gamma_{\breve{M}}\left(n \omega_{1}+m(1-n) \omega_{2}\right) \subseteq \gamma_{\breve{M}}\left(\omega_{1}\right) \cup \gamma_{\breve{M}}\left(\omega_{2}\right) \\
& \gamma_{\breve{N}}\left(n \omega_{1}+m(1-n) \omega_{2}\right) \subseteq \gamma_{\breve{N}}\left(\omega_{1}\right) \cup \gamma_{\breve{N}}\left(\omega_{2}\right)
\end{aligned}
$$

and hence,

$$
\gamma_{\breve{W}}\left(n \omega_{1}+m(1-n) \omega_{2}\right) \subseteq\left(\gamma_{\breve{M}}\left(\omega_{1}\right) \cup \gamma_{\breve{M}}\left(\omega_{2}\right)\right) \cup\left(\gamma_{\breve{N}}\left(\omega_{1}\right) \cup \gamma_{\breve{N}}\left(\omega_{2}\right)\right)
$$
and thus

$$
\gamma_{\breve{W}}\left(n \omega_{1}+m(1-n) \omega_{2}\right) \subseteq \gamma_{\breve{W}}\left(\omega_{1}\right) \cup \gamma_{\breve{W}}\left(\omega_{2}\right)
$$

Theorem 3.6. $\Gamma_{\breve{M}} \cap \Gamma_{\breve{N}}$ is $(m, n)$-concave FSS when both $\Gamma_{\breve{M}}$ and $\Gamma_{\breve{N}}$ are $(m, n)$-concave FSSs. 
Proof. Suppose that for $\omega_{1}, \omega_{2} \in \breve{L}$, and $n \in \breve{J}$ and $\Gamma_{\breve{W}}=\Gamma_{\breve{M}} \cap \Gamma_{\breve{N}}$. Then,

$$
\gamma_{\breve{W}}\left(n \omega_{1}+m(1-n) \omega_{2}\right)=\gamma_{\breve{M}}\left(n \omega_{1}+m(1-n) \omega_{2}\right) \cap \gamma_{\breve{N}}\left(n \omega_{1}+m(1-n) \omega_{2}\right)
$$

Now, since $\Gamma_{\breve{M}}$ and $\Gamma_{\breve{N}}$ are $(m, n)$-concave,

$$
\begin{aligned}
& \gamma_{\breve{M}}\left(n \omega_{1}+m(1-n) \omega_{2}\right) \subseteq \gamma_{\breve{M}}\left(\omega_{1}\right) \cup \gamma_{\breve{M}}\left(\omega_{2}\right) \\
& \gamma_{\breve{N}}\left(n \omega_{1}+m(1-n) \omega_{2}\right) \subseteq \gamma_{\breve{N}}\left(\omega_{1}\right) \cup \gamma_{\breve{N}}\left(\omega_{2}\right)
\end{aligned}
$$

and hence,

$$
\gamma_{\breve{W}}\left(n \omega_{1}+m(1-n) \omega_{2}\right) \subseteq\left(\gamma_{\breve{M}}\left(\omega_{1}\right) \cup \gamma_{\breve{M}}\left(\omega_{2}\right)\right) \cap\left(\gamma_{\breve{N}}\left(\omega_{1}\right) \cup \gamma_{\breve{N}}\left(\omega_{2}\right)\right)
$$
and thus

$$
\gamma_{\breve{W}}\left(n \omega_{1}+m(1-n) \omega_{2}\right) \subseteq \gamma_{\breve{W}}\left(\omega_{1}\right) \cap \gamma_{\breve{W}}\left(\omega_{2}\right)
$$

Theorem 3.7. $\Gamma_{\breve{A}}^{c}$ is $(m, n)$-concave FSS when $\Gamma_{\breve{A}}$ is $(m, n)$-convex FSS.

Proof. Suppose that for $\omega_{1}, \omega_{2} \in \breve{L}, n \in \breve{J}$, and $\Gamma_{\breve{A}}$ be $(m, n)$-convex FSS.

Since $\Gamma_{\breve{A}}$ is $(m, n)$-convex,

$$
\gamma_{\breve{A}}\left(n \omega_{1}+m(1-n) \omega_{2}\right) \supseteq \gamma_{\breve{A}}\left(\omega_{1}\right) \cap \gamma_{\breve{A}}\left(\omega_{2}\right)
$$

or

$$
\breve{U} \backslash \gamma_{\breve{A}}\left(n \omega_{1}+m(1-n) \omega_{2}\right) \subseteq \breve{U} \backslash\left\{\gamma_{\breve{A}}\left(\omega_{1}\right) \cap \gamma_{\breve{A}}\left(\omega_{2}\right)\right\}
$$

If $\gamma_{\breve{A}}\left(\omega_{1}\right) \supset \gamma_{\breve{A}}\left(\omega_{2}\right)$ then $\gamma_{\breve{A}}\left(\omega_{1}\right) \cap \gamma_{\breve{A}}\left(\omega_{2}\right)=\gamma_{\breve{A}}\left(\omega_{2}\right)$

then,

$$
\breve{U} \backslash \gamma_{\breve{A}}\left(n \omega_{1}+m(1-n) \omega_{2}\right) \subseteq \breve{U} \backslash \gamma_{\breve{A}}\left(\omega_{2}\right)
$$

If $\gamma_{\breve{A}}\left(\omega_{1}\right) \subset \gamma_{\breve{A}}\left(\omega_{2}\right)$ then $\gamma_{\breve{A}}\left(\omega_{1}\right) \cap \gamma_{\breve{A}}\left(\omega_{2}\right)=\gamma_{\breve{A}}\left(\omega_{1}\right)$

then we may write

$$
\breve{U} \backslash \gamma_{\breve{A}}\left(n \omega_{1}+m(1-n) \omega_{2}\right) \subseteq \breve{U} \backslash \gamma_{\breve{A}}\left(\omega_{1}\right)
$$

so we have

$$
\breve{U} \backslash \gamma_{\breve{A}}\left(n \omega_{1}+m(1-n) \omega_{2}\right) \subseteq\left\{\breve{U} \backslash \gamma_{\breve{A}}\left(\omega_{1}\right) \cup \breve{U} \backslash \gamma_{\breve{A}}\left(\omega_{2}\right)\right\} .
$$

which shows that $\Gamma_{\breve{A}}^{c}$ is $(m, n)$-concave FSS.

Theorem 3.8. $\Gamma_{\breve{A}}^{c}$ is $(m, n)$-convex FSS when $\Gamma_{\breve{A}}$ is $(m, n)$-concave FSS.

Proof. Suppose that for $\omega_{1}, \omega_{2} \in \breve{L}, n \in \breve{J}$, and $\Gamma_{\breve{A}}$ be $(m, n)$-concave FSS.

Since $\Gamma_{\breve{A}}$ is $(m, n)$-concave,

$$
\gamma_{\breve{A}}\left(n \omega_{1}+m(1-n) \omega_{2}\right) \subseteq \gamma_{\breve{A}}\left(\omega_{1}\right) \cup \gamma_{\breve{A}}\left(\omega_{2}\right)
$$

or

$$
\breve{U} \backslash \gamma_{\breve{A}}\left(n \omega_{1}+m(1-n) \omega_{2}\right) \supseteq \breve{U} \backslash\left\{\gamma_{\breve{A}}\left(\omega_{1}\right) \cup \gamma_{\breve{A}}\left(\omega_{2}\right)\right\}
$$

If $\gamma_{\breve{A}}\left(\omega_{1}\right) \supset \gamma_{\breve{A}}\left(\omega_{2}\right)$ then $\gamma_{\breve{A}}\left(\omega_{1}\right) \cup \gamma_{\breve{A}}\left(\omega_{2}\right)=\gamma_{\breve{A}}\left(\omega_{1}\right)$

then,

$$
\breve{U} \backslash \gamma_{\breve{A}}\left(n \omega_{1}+m(1-n) \omega_{2}\right) \supseteq \breve{U} \backslash \gamma_{\breve{A}}\left(\omega_{1}\right)
$$


If $\gamma_{\breve{A}}\left(\omega_{1}\right) \subset \gamma_{\breve{A}}\left(\omega_{2}\right)$ then $\gamma_{\breve{A}}\left(\omega_{1}\right) \cup \gamma_{\breve{A}}\left(\omega_{2}\right)=\gamma_{\breve{A}}\left(\omega_{2}\right)$

then we may write

$$
\breve{U} \backslash \gamma_{\breve{A}}\left(n \omega_{1}+m(1-n) \omega_{2}\right) \supseteq \breve{U} \backslash \gamma_{\breve{A}}\left(\omega_{2}\right)
$$

so we have

$$
\breve{U} \backslash \gamma_{\breve{A}}\left(n \omega_{1}+m(1-n) \omega_{2}\right) \subseteq\left\{\breve{U} \backslash \gamma_{\breve{A}}\left(\omega_{1}\right) \cup \breve{U} \backslash \gamma_{\breve{A}}\left(\omega_{2}\right)\right\}
$$

So, $\Gamma_{\breve{A}}^{c}$ is $(m, n)$-convex FSS.

Theorem 3.9. $\Gamma_{\breve{A}}$ is $(m, n)$-concave FSS on $\breve{L}$ iff for every $n \in[0,1]$ and $\breve{\delta} \in \breve{P}(\breve{U})$, $\Gamma_{\breve{A}}^{\breve{\delta}}$ is $(m, n)$-concave FSS on $\breve{L}$.

Proof. Suppose that $\Gamma_{\breve{A}}$ is $(m, n)$-concave FSS.If $\omega_{1}, \omega_{2} \in \breve{L}$ and $\breve{\delta} \in \breve{P}(\breve{U})$, then $\gamma_{\breve{A}}\left(\omega_{1}\right) \supseteq \breve{\delta}$ and $\gamma_{\breve{A}}\left(\omega_{2}\right) \supseteq \breve{\delta}$ then $\gamma_{\breve{A}}\left(\omega_{1}\right) \cup \gamma_{\breve{A}}\left(\omega_{2}\right) \supseteq \breve{\delta}$. It follows from $(m, n)-$ concavity of $\Gamma_{\breve{A}}$ that

$$
\breve{\delta} \subseteq \gamma_{\breve{A}}\left(\omega_{1}\right) \cap \gamma_{\breve{A}}\left(\omega_{2}\right) \subseteq \gamma_{\breve{A}}\left(n \omega_{1}+m(1-n) \omega_{2}\right) \subseteq \gamma_{\breve{A}}\left(\omega_{1}\right) \cup \gamma_{\breve{A}}\left(\omega_{2}\right)
$$

SO

$$
\breve{\delta} \subseteq \gamma_{\breve{A}}\left(n \omega_{1}+m(1-n) \omega_{2}\right)
$$

therefore

$\Gamma_{\breve{A}}^{\breve{\delta}}$ is a concave FSS.

Conversely suppose that $\Gamma_{\breve{A}}^{\breve{\delta}}$ is $(m, n)$-concave FSS for every $n \in[0,1]$. For $\omega_{1}, \omega_{2} \in \breve{L}$, $\Gamma_{\breve{A}}^{\breve{\delta}}$ is concave with $\breve{\delta}=\gamma_{\breve{A}}\left(\omega_{1}\right) \cup \gamma_{\breve{A}}\left(\omega_{2}\right)$. Since $\gamma_{\breve{A}}\left(\omega_{1}\right) \subseteq \breve{\delta}$ and $\gamma_{\breve{A}}\left(\omega_{2}\right) \subseteq \breve{\delta}$, we have $\omega_{1} \in \Gamma_{\breve{A}}^{\breve{\delta}}$ and $\omega_{2} \in \Gamma_{\breve{A}}^{\breve{\delta}}$, so $n \omega_{1}+m(1-n) \omega_{2} \in \Gamma_{\breve{A}}^{\breve{\delta}}$.

implies

$$
\gamma_{\breve{A}}\left(n \omega_{1}+m(1-n) \omega_{2}\right) \subseteq \Gamma_{\breve{A}}^{\breve{\delta}}
$$

therefore, $\gamma_{\breve{A}}\left(n \omega_{1}+m(1-n) \omega_{2}\right) \subseteq \gamma_{\breve{A}}\left(\omega_{1}\right) \cup \gamma_{\breve{A}}\left(\omega_{2}\right)$, which proves the $(m, n)$ concavity of $\Gamma_{\breve{A}}$ on $\breve{L}$.

\section{4. $(m, n)$-Convex And $(m, n)$-Concave Fuzzy Soft Sets In First And Second SENSE}

In this section, $(m, n)$-Convex and $(m, n)$-concave FSSs are defined in $1^{\text {st }}$ and $2^{\text {nd }}$ sense and then some desired results are proved.

Definition 15. The fuzzy soft set $\Gamma_{\breve{A}}$ on $\breve{L}$ is called a $(m, n)$-convex FSS in $1^{\text {st }}$ sense if

$$
\gamma_{\breve{A}}\left(n \omega_{1}+m\left(1-n^{\eta}\right) \omega_{2}\right) \supseteq \gamma_{\breve{A}}\left(\omega_{1}\right) \tilde{\cap} \gamma_{\breve{A}}\left(\omega_{2}\right)
$$

for $\omega_{1}, \omega_{2} \in \breve{L}, m \in \breve{J}$ and $\eta, n \in(0,1]$. 
Definition 16. The fuzzy soft set $\Gamma_{\breve{A}}$ on $\breve{L}$ is called a $(m, n)$-convex FSS in $2^{n d}$ sense if

$$
\gamma_{\breve{A}}\left(n \omega_{1}+m(1-n)^{\eta} \omega_{2}\right) \supseteq \gamma_{\breve{A}}\left(\omega_{1}\right) \tilde{\cap} \gamma_{\breve{A}}\left(\omega_{2}\right)
$$

for $\omega_{1}, \omega_{2} \in \breve{L}, m \in \breve{J}$ and $\eta, n \in(0,1]$.

Theorem 4.1. $\Gamma_{\breve{M}} \cap \Gamma_{\breve{N}}$ is a $(m, n)$-convex $F S S$ when both $\Gamma_{\breve{M}}$ and $\Gamma_{\breve{N}}$ are $(m, n)$-convex FSSs in the $1^{\text {st }}$ sense.

Proof. Suppose that for $\omega_{1}, \omega_{2} \in \breve{L}$, and $\Gamma_{\breve{W}}=\Gamma_{\breve{M}} \cap \Gamma_{\breve{N}}$, Then,

$$
\gamma_{\breve{W}}\left(n \omega_{1}+m\left(1-n^{\eta}\right) \omega_{2}\right)=\gamma_{\breve{M}}\left(n \omega_{1}+m\left(1-n^{\eta}\right) \omega_{2}\right) \cap \gamma_{\breve{N}}\left(n \omega_{1}+m\left(1-n^{\eta}\right) \omega_{2}\right)
$$

Now, since $\Gamma_{\breve{M}}$ and $\Gamma_{\breve{N}}$ are $(m, n)$-convex in the $1^{\text {st }}$ sense,

$$
\begin{aligned}
& \gamma_{\breve{M}}\left(n \omega_{1}+m\left(1-n^{\eta}\right) \omega_{2}\right) \supseteq \gamma_{\breve{M}}\left(\omega_{1}\right) \cap \gamma_{\breve{M}}\left(\omega_{2}\right) \\
& \gamma_{\breve{N}}\left(n \omega_{1}+m\left(1-n^{\eta}\right) \omega_{2}\right) \supseteq \gamma_{\breve{N}}\left(\omega_{1}\right) \cap \gamma_{\breve{N}}\left(\omega_{2}\right)
\end{aligned}
$$

which implies

$$
\gamma_{\breve{W}}\left(n \omega_{1}+m\left(1-n^{\eta}\right) \omega_{2}\right) \supseteq\left(\gamma_{\breve{M}}\left(\omega_{1}\right) \cap \gamma_{\breve{M}}\left(\omega_{2}\right)\right) \cap\left(\gamma_{\breve{N}}\left(\omega_{1}\right) \cap \gamma_{\breve{N}}\left(\omega_{2}\right)\right)
$$
and thus

$$
\gamma_{\breve{W}}\left(n \omega_{1}+m\left(1-n^{\eta}\right) \omega_{2}\right) \supseteq \gamma_{\breve{W}}(\omega) \cap \gamma_{\breve{W}}\left(\omega_{2}\right)
$$

Theorem 4.2. $\Gamma_{\breve{M}} \cap \Gamma_{\breve{N}}$ is a $(m, n)$-convex FSS in the $2^{\text {nd }}$ sense when both $\Gamma_{\breve{M}}$ and $\Gamma_{\breve{N}}$ are $(m, n)$-convex FSSs in the $2^{\text {nd }}$ sense.

Proof. Suppose that for $\omega_{1}, \omega_{2} \in \breve{L}$, and $\Gamma_{\breve{W}}=\Gamma_{\breve{M}} \cap \Gamma_{\breve{N}}$, Then,

$$
\gamma_{\breve{W}}\left(n \omega_{1}+m(1-n)^{\eta} \omega_{2}\right)=\gamma_{\breve{M}}\left(n \omega_{1}+m(1-n)^{\eta} \omega_{2}\right) \cap \gamma_{\breve{N}}\left(n \omega_{1}+m(1-n)^{\eta} \omega_{2}\right)
$$

Now, since $\Gamma_{\breve{M}}$ and $\Gamma_{\breve{N}}$ are $(m, n)$-convex in the $2^{\text {nd }}$ sense,

$$
\begin{aligned}
& \gamma_{\breve{M}}\left(n \omega_{1}+m(1-n)^{\eta} \omega_{2}\right) \supseteq \gamma_{\breve{M}}\left(\omega_{1}\right) \cap \gamma_{\breve{M}}\left(\omega_{2}\right) \\
& \gamma_{\breve{N}}\left(n \omega_{1}+m(1-n)^{\eta} \omega_{2}\right) \supseteq \gamma_{\breve{N}}\left(\omega_{1}\right) \cap \gamma_{\breve{N}}\left(\omega_{2}\right)
\end{aligned}
$$

which implies

$$
\gamma_{\breve{W}}\left(n \omega_{1}+m(1-n)^{\eta} \omega_{2}\right) \supseteq\left(\gamma_{\breve{M}}\left(\omega_{1}\right) \cap \gamma_{\breve{M}}\left(\omega_{2}\right)\right) \cap\left(\gamma_{\breve{N}}\left(\omega_{1}\right) \cap \gamma_{\breve{N}}\left(\omega_{2}\right)\right)
$$
and thus

$$
\gamma_{\breve{W}}\left(n \omega_{1}+m(1-n)^{\eta} \omega_{2}\right) \supseteq \gamma_{\breve{W}}(\omega) \cap \gamma_{\breve{W}}\left(\omega_{2}\right)
$$


Theorem 4.3. $\Gamma_{\breve{A}}$ is a $(m, n)$-convex FSS in the $1^{\text {st }}$ sense on $\breve{L}$ iff for every $n \in[0,1]$ and $\breve{\delta} \in \breve{P}(\breve{U}), \Gamma_{\breve{A}}^{\breve{\delta}}$ is a $(m, n)$-convex FSS in the $1^{\text {st }}$ sense on $\breve{L}$.

Proof. Suppose $\Gamma_{\breve{A}}$ is a $(m, n)$-convex FSS in the $1^{\text {st }}$ sense. If $\omega_{1}, \omega_{2} \in \breve{L}$, and $\breve{\delta} \in$ $\breve{P}(\breve{U})$, then $\gamma_{\breve{A}}\left(\omega_{1}\right) \supseteq \breve{\delta}$ and $\gamma_{\breve{A}}\left(\omega_{2}\right) \supseteq \breve{\delta}$. It follows from $(m, n)$-convexity of $\Gamma_{\breve{A}}$ that

$$
\gamma_{\breve{A}}\left(n \omega_{1}+m\left(1-n^{\eta}\right) \omega_{2}\right) \supseteq \gamma_{\breve{A}}\left(\omega_{1}\right) \cap \gamma_{\breve{A}}\left(\omega_{2}\right)
$$

and thus $\Gamma_{\breve{A}}^{\breve{\delta}}$ is a $(m, n)$-convex FSS in the $1^{\text {st }}$ sense.

Conversely suppose that $\Gamma_{\breve{A}}^{\breve{\delta}}$ is a $(m, n)$-convex FSS in the $1^{\text {st }}$ sense for every $n \in[0,1]$. For $\omega_{1}, \omega_{2} \in \breve{L}, \Gamma_{\breve{A}}^{\breve{\delta}}$ is $(m, n)$-convex for $\breve{\delta}=\gamma_{\breve{A}}\left(\omega_{1}\right) \cap \gamma_{\breve{A}}\left(\omega_{2}\right)$. Since $\gamma_{\breve{A}}\left(\omega_{1}\right) \supseteq \breve{\delta}$ and $\gamma_{\breve{A}}\left(\omega_{2}\right) \supseteq \breve{\delta}$, we have $\omega_{1} \in \Gamma_{\breve{A}}^{\breve{\delta}}$ and $\omega_{2} \in \Gamma_{\breve{A}}^{\breve{\delta}}$, hence $n \omega_{1}+m\left(1-n^{\eta}\right) \omega_{2} \in$ $\Gamma_{\breve{A}}^{\breve{\delta}}$. Therefore, $\gamma_{\breve{A}}\left(n \omega_{1}+m\left(1-n^{\eta}\right) \omega_{2}\right) \supseteq \gamma_{\breve{A}}\left(\omega_{1}\right) \cap \gamma_{\breve{A}}\left(\omega_{2}\right)$, which indicates $\Gamma_{\breve{M}}$ is a $(m, n)$-convex FSS in the $1^{\text {st }}$ sense on $\breve{L}$.

Theorem 4.4. $\Gamma_{\breve{A}}$ is a $(m, n)$-convex FSS in the $2^{\text {nd }}$ sense on $\breve{L}$ iff for every $n \in[0,1]$ and $\breve{\delta} \in \breve{P}(\breve{U}), \Gamma_{\breve{A}}^{\breve{\delta}}$ is a $(m, n)$-convex FSS in the $2^{\text {nd }}$ sense on $\breve{L}$.

Proof. Suppose $\Gamma_{\breve{A}}$ is a $(m, n)$-convex FSS in the $2^{\text {nd }}$ sense. If $\omega_{1}, \omega_{2} \in \breve{L}$ and $\breve{\delta} \in$ $\breve{P}(\breve{U})$, then $\gamma_{\breve{A}}\left(\omega_{1}\right) \supseteq \breve{\delta}$ and $\gamma_{\breve{A}}\left(\omega_{2}\right) \supseteq \breve{\delta}$. It follows from the $(m, n)$-convexity of $\Gamma_{\breve{A}}$ that

$$
\gamma_{\breve{A}}\left(n \omega_{1}+m(1-n)^{\eta} \omega_{2}\right) \supseteq \gamma_{\breve{A}}\left(\omega_{1}\right) \cap \gamma_{\breve{A}}\left(\omega_{2}\right)
$$

and thus $\Gamma_{\breve{A}}^{\breve{\delta}}$ is a $(m, n)$-convex FSS in the $2^{n d}$ sense.

Conversly suppose that $\Gamma_{\breve{A}}^{\breve{\delta}}$ is a $(m, n)$-convex FSS in the $2^{\text {nd }}$ sense for every $\rho \in[0,1]$. For $\omega_{1}, \omega_{2} \in \breve{L}, \Gamma_{\breve{A}}^{\breve{\delta}}$ is $(m, n)$-convex for $\breve{\delta}=\gamma_{\breve{A}}\left(\omega_{1}\right) \cap \gamma_{\breve{A}}\left(\omega_{2}\right)$. Since $\gamma_{\breve{A}}\left(\omega_{1}\right) \supseteq \breve{\delta}$ and $\gamma_{\breve{A}}\left(\omega_{2}\right) \supseteq \breve{\delta}$, we have $\omega_{1} \in \breve{A}^{\breve{\delta}}$ and $\omega_{2} \in \Gamma_{\breve{A}}^{\breve{\delta}}$, hence $n \omega_{1}+m(1-n)^{\eta} \omega_{2} \in \Gamma_{\breve{A}}^{\breve{\delta}}$. Therefore, $\gamma_{\breve{A}}\left(n \omega_{1}+m(1-n)^{\eta} \omega_{2}\right) \supseteq \gamma_{\breve{A}}\left(\omega_{1}\right) \cap \gamma_{\breve{A}}\left(\omega_{2}\right)$, which indicates $\Gamma_{\breve{A}}$ is a $(m, n)$-convex FSS in the $2^{\text {nd }}$ sense on $\breve{L}$.

Definition 17. The fuzzy soft set $\Gamma_{\breve{A}}$ on $\breve{L}$ is called a $(m, n)$-concave FSS in the $1^{s t}$ sense if

$$
\gamma_{\breve{A}}\left(n \omega_{1}+m\left(1-n^{\eta}\right) \omega_{2}\right) \subseteq \gamma_{\breve{A}}\left(\omega_{1}\right) \cup \gamma_{\breve{A}}\left(\omega_{2}\right)
$$

for every $\omega_{1}, \omega_{2} \in \breve{L}, n \in \breve{J}$.

Definition 18. The fuzzy soft set $\Gamma_{\breve{A}}$ on $\breve{L}$ is called a $(m, n)$-concave FSS in the $2^{\text {nd }}$ sense if

$$
\gamma_{\breve{A}}\left(n \omega_{1}+m(1-n)^{\eta} \omega_{2}\right) \subseteq \gamma_{\breve{A}}\left(\omega_{1}\right) \cup \gamma_{\breve{A}}\left(\omega_{2}\right)
$$

for every $\omega_{1}, \omega_{2} \in \breve{L}, n \in \breve{J}$. 
Theorem 4.5. $\Gamma_{\breve{M}} \cap \Gamma_{\breve{N}}$ is a $(m, n)$-concave FSS in the $1^{\text {st }}$ sense when both $\Gamma_{\breve{M}}$ and $\Gamma_{\breve{N}}$ are $(m, n)$-concave FSSs in the $1^{\text {st }}$ sense.

Proof. Suppose that for $\omega_{1}, \omega_{2} \in \breve{L}$, and $n \in \breve{J}$ and $\Gamma_{\breve{W}}=\Gamma_{\breve{M}} \cap \Gamma_{\breve{N}}$. Then,

$$
\gamma_{\breve{W}}\left(n \omega_{1}+m\left(1-n^{\eta}\right) \omega_{2}\right)=\gamma_{\breve{M}}\left(n \omega_{1}+m\left(1-n^{\eta}\right) \omega_{2}\right) \cap \gamma_{\breve{N}}\left(n \omega_{1}+m\left(1-n^{\eta}\right) \omega_{2}\right)
$$

Now, since $\Gamma_{\breve{M}}$ and $\Gamma_{\breve{N}}$ are $(m, n)$-concave in the $1^{\text {st }}$ sense,

$$
\begin{aligned}
& \gamma_{\breve{M}}\left(n \omega_{1}+m\left(1-n^{\eta}\right) \omega_{2}\right) \subseteq \gamma_{\breve{M}}\left(\omega_{1}\right) \cup \gamma_{\breve{M}}\left(\omega_{2}\right) \\
& \gamma_{\breve{N}}\left(n \omega_{1}+m\left(1-n^{\eta}\right) \omega_{2}\right) \subseteq \gamma_{\breve{N}}\left(\omega_{1}\right) \cup \gamma_{\breve{N}}\left(\omega_{2}\right)
\end{aligned}
$$

and hence,

$$
\gamma_{\breve{W}}\left(n \omega_{1}+m\left(1-n^{\eta}\right) \omega_{2}\right) \subseteq\left(\gamma_{\breve{M}}\left(\omega_{1}\right) \cup \gamma_{\breve{M}}\left(\omega_{2}\right)\right) \cap\left(\gamma_{\breve{N}}\left(\omega_{1}\right) \cup \gamma_{\breve{N}}\left(\omega_{2}\right)\right)
$$
and thus

$$
\gamma_{\breve{W}}\left(n \omega_{1}+m\left(1-n^{\eta}\right) \omega_{2}\right) \subseteq \gamma_{\breve{W}}\left(\omega_{1}\right) \cup \gamma_{\breve{W}}\left(\omega_{2}\right)
$$

Theorem 4.6. $\Gamma_{\breve{M}} \cap \Gamma_{\breve{N}}$ is a $(m, n)$-concave FSS in the $2^{\text {nd }}$ sense when both $\Gamma_{\breve{M}}$ and $\Gamma_{\breve{N}}$ are $(m, n)$-concave FSSs in the $2^{\text {nd }}$ sense.

Proof. Suppose that for $\omega_{1}, \omega_{2} \in \breve{L}$, and $n \in \breve{J}$ and $\Gamma_{\breve{W}}=\Gamma_{\breve{M}} \cap \Gamma_{\breve{N}}$. Then,

$$
\gamma_{\breve{W}}\left(n \omega_{1}+m(1-n)^{\eta} \omega_{2}\right)=\gamma_{\breve{M}}\left(n \omega_{1}+m(1-n)^{\eta} \omega_{2}\right) \cap \gamma_{\breve{N}}\left(n \omega_{1}+m(1-n)^{\eta} \omega_{2}\right)
$$

Now, since $\Gamma_{\breve{M}}$ and $\Gamma_{\breve{N}}$ are $(m, n)$-concave in the $2^{\text {nd }}$ sense,

$$
\begin{aligned}
& \gamma_{\breve{M}}\left(n \omega_{1}+m(1-n)^{\eta} \omega_{2}\right) \subseteq \gamma_{\breve{M}}\left(\omega_{1}\right) \cup \gamma_{\breve{M}}\left(\omega_{2}\right) \\
& \gamma_{\breve{N}}\left(n \omega_{1}+m(1-n)^{\eta} \omega_{2}\right) \subseteq \gamma_{\breve{N}}\left(\omega_{1}\right) \cup \gamma_{\breve{N}}\left(\omega_{2}\right)
\end{aligned}
$$

and hence,

$$
\gamma_{\breve{W}}\left(n \omega_{1}+m(1-n)^{\eta} \omega_{2}\right) \subseteq\left(\gamma_{\breve{M}}\left(\omega_{1}\right) \cup \gamma_{\breve{M}}\left(\omega_{2}\right)\right) \cap\left(\gamma_{\breve{N}}\left(\omega_{1}\right) \cup \gamma_{\breve{N}}\left(\omega_{2}\right)\right)
$$

and thus

$$
\gamma_{\breve{W}}\left(n \omega_{1}+m(1-n)^{\eta} \omega_{2}\right) \subseteq \gamma_{\breve{W}}\left(\omega_{1}\right) \cup \gamma_{\breve{W}}\left(\omega_{2}\right)
$$

Theorem 4.7. $\Gamma_{\breve{M}}^{c}$ is a $(m, n)$-concave FSS in the $2^{\text {nd }}$ sense when $\Gamma_{\breve{M}}$ is a $(m, n)$-convex FSS in the $2^{\text {nd }}$ sense.

Proof. Suppose that for $\omega_{1}, \omega_{2} \in \breve{L}, n \in \breve{J}$, and $\Gamma_{\breve{M}}$ be a $(m, n)$-convex FSS in the $2^{n d}$ sense.

then, since $\Gamma_{\breve{M}}$ is $(m, n)$-convex in the $2^{\text {nd }}$ sense,

$$
\gamma_{\breve{M}}\left(n \omega_{1}+m(1-n)^{\eta} \omega_{2}\right) \supseteq \gamma_{\breve{M}}\left(\omega_{1}\right) \cap \gamma_{\breve{M}}\left(\omega_{2}\right)
$$

or

$$
\breve{U} \backslash \gamma_{\breve{M}}\left(n \omega_{1}+m(1-n)^{\eta} \omega_{2}\right) \subseteq \breve{U} \backslash\left\{\gamma_{\breve{M}}\left(\omega_{1}\right) \cap \gamma_{\breve{M}}\left(\omega_{2}\right)\right\}
$$


If $\gamma_{\breve{M}}\left(\omega_{1}\right) \supset \gamma_{\breve{M}}\left(\omega_{2}\right)$ then we may write

$$
\breve{U} \backslash \gamma_{\breve{M}}\left(n \omega_{1}+m(1-n)^{\eta} \omega_{2}\right) \subseteq \breve{U} \backslash \gamma_{\breve{M}}\left(\omega_{2}\right)
$$

If $\gamma_{\breve{M}}\left(\omega_{1}\right) \subset \gamma_{\breve{M}}\left(\omega_{2}\right)$ then we may write

$$
\breve{U} \backslash \gamma_{\breve{M}}\left(n \omega_{1}+m(1-n)^{\eta} \omega_{2}\right) \subseteq U \backslash \gamma_{\breve{M}}\left(\omega_{1}\right)
$$

From the above equations, we have

$$
\breve{U} \backslash \gamma_{\breve{M}}\left(n \omega_{1}+m(1-n)^{\eta} \omega_{2}\right) \subseteq\left(U \backslash \gamma_{\breve{M}}\left(\omega_{1}\right)\right) \cup\left(\breve{U} \backslash \gamma_{\breve{M}}\left(\omega_{2}\right)\right)
$$

So, $\Gamma_{\breve{M}}^{c}$ is a $(m, n)$-concave FSS in the $2^{n d}$ sense.

Theorem 4.8. $\Gamma_{\breve{M}}^{c}$ is a $(m, n)$-convex FSS in the $1^{\text {st }}$ sense when $\Gamma_{\breve{M}}$ is a $(m, n)$-concave FSS in the $1^{\text {st }}$ sense.

Proof. Suppose that there exist $\omega_{1}, \omega_{2} \in \breve{L}, n \in \breve{J}$ and $\Gamma_{\breve{M}}$ be a $(m, n)$-concave FSS in the $1^{\text {st }}$ sense.

then, since $\Gamma_{\breve{M}}$ is $(m, n)$-concave in the $1^{\text {st }}$ sense,

$$
\gamma_{\breve{M}}\left(n \omega_{1}+m\left(1-n^{\eta}\right) \omega_{2}\right) \subseteq \gamma_{\breve{M}}\left(\omega_{1}\right) \cup \gamma_{\breve{M}}\left(\omega_{2}\right)
$$

or

$$
\breve{U} \backslash \gamma_{\breve{M}}\left(n \omega_{1}+m\left(1-n^{\eta}\right) \omega_{2}\right) \supseteq U \backslash\left\{\gamma_{\breve{M}}\left(\omega_{1}\right) \cup \gamma_{\breve{M}}\left(\omega_{2}\right)\right\}
$$

If $\gamma_{\breve{M}}\left(\omega_{1}\right) \supset \gamma_{\breve{M}}\left(\omega_{2}\right)$ then we may write

$$
\breve{U} \backslash \gamma_{\breve{M}}\left(n \omega_{1}+m\left(1-n^{\eta}\right) \omega_{2}\right) \supseteq U \backslash \gamma_{\breve{M}}\left(\omega_{1}\right) \text {. }
$$

If $\gamma_{\breve{M}}\left(\omega_{1}\right) \subset \gamma_{\breve{M}}\left(\omega_{2}\right)$ then we may write

$$
\breve{U} \backslash \gamma_{\breve{M}}\left(n \omega_{1}+m\left(1-n^{\eta}\right) \omega_{2}\right) \supseteq U \backslash \gamma_{\breve{M}}\left(\omega_{2}\right) .
$$

From (24) and (25), we have

$$
\breve{U} \backslash \gamma_{\breve{M}}\left(n \omega_{1}+m\left(1-n^{\eta}\right) \omega_{2}\right) \supseteq\left(U \backslash \gamma_{\breve{M}}\left(\omega_{1}\right)\right) \cap\left(U \backslash \gamma_{\breve{M}}\left(\omega_{2}\right)\right) .
$$

So, $\Gamma_{\breve{M}}^{c}$ is a $(m, n)$-convex FSS in the $1^{s t}$ sense.

Theorem 4.9. $\Gamma_{\breve{M}}$ is a $(m, n)$-concave FSS in the $1^{\text {st }}$ sense on $\breve{L}$ if and only if for every $n \in[0,1]$ and $\breve{\delta} \in \breve{P}(\breve{U}), \Gamma_{\breve{M}}^{\breve{\delta}}$ is a $(m, n)$-concave FSS in the $1^{\text {st }}$ sense on $\breve{L}$.

Proof. Suppose $\Gamma_{\breve{M}}$ is a $(m, n)$-concave FSS in the $1^{\text {st }}$ sense. If $\omega_{1}, \omega_{2} \in \breve{L}$ and $\breve{\delta} \in$ $\breve{P}(\breve{U})$,then $\gamma_{\breve{M}}\left(\omega_{1}\right) \supseteq \breve{\delta}$ and $\gamma_{\breve{M}}\left(\omega_{2}\right) \supseteq \breve{\delta}$. It follows from the $(m, n)$-concavity of $\Gamma_{\breve{M}}$ in the $1^{\text {st }}$ sense that

$$
\gamma_{\breve{M}}\left(n \omega_{1}+m\left(1-n^{\eta}\right) \omega_{2}\right) \subseteq \gamma_{\breve{M}}\left(\omega_{1}\right) \cup \gamma_{\breve{M}}\left(\omega_{2}\right)
$$

and thus $\Gamma_{\breve{M}}^{\breve{\delta}}$ is a $(m, n)$-concave FSS in the $1^{\text {st }}$ sense.

Conversely suppose that $\Gamma_{\breve{M}}^{\breve{\delta}}$ is a $(m, n)$-concave FSS in the $1^{\text {st }}$ sense for every $\rho \in[0,1]$. For $\omega_{1}, \omega_{2} \in \breve{L}, \Gamma_{\breve{M}}^{\breve{\delta}}$ is $(m, n)$-concave FSS for $\breve{\delta}=\gamma_{\breve{M}}\left(\omega_{1}\right) \cup \gamma_{\breve{M}}\left(\omega_{2}\right)$. Since $\gamma_{\breve{M}}\left(\omega_{1}\right) \subseteq$ $\breve{\delta}$ and $\gamma_{\breve{M}}\left(\omega_{2}\right) \subseteq \breve{\delta}$, we have $\omega_{1} \in \Gamma_{\breve{M}}^{\breve{\delta}}$ and $\omega_{2} \in \Gamma_{\breve{M}}^{\breve{\delta}}$, hence $n \omega_{1}+m\left(1-n^{\eta}\right) \omega_{2} \in$ 
$\Gamma_{\breve{M}}^{\breve{\delta}}$.Therefore, $\gamma_{\breve{M}}\left(n \omega_{1}+m\left(1-n^{\eta}\right) \omega_{2}\right) \subseteq \breve{\delta}=\gamma_{\breve{M}}\left(\omega_{1}\right) \cup \gamma_{\breve{M}}\left(\omega_{2}\right)$, which indicates $\Gamma_{\breve{M}}$ is a $(m, n)$-concave FSS in the $1^{s t}$ sense on $\breve{L}$.

Theorem 4.10. $\Gamma_{\breve{M}}$ is a $(m, n)$-concave FSS in the $2^{\text {nd }}$ sense on $\breve{L}$ if and only if for every $n \in[0,1]$ and $\breve{\delta} \in \breve{P}(\breve{U}), \Gamma_{\breve{M}}^{\breve{s}}$ is a $(m, n)$-concave FSS in the $2^{\text {nd }}$ sense on $\breve{L}$.

Proof. Suppose that $\Gamma_{\breve{M}}$ is a ( $\left.m, n\right)$-concave FSS in the $2^{\text {nd }}$ sense. If $\omega_{1}, \omega_{2} \in \breve{L}$ and $\breve{\delta} \in \breve{P}(\breve{U})$,then $\gamma_{\breve{M}}\left(\omega_{1}\right) \supseteq \breve{\delta}$ and $\gamma_{\breve{M}}\left(\omega_{2}\right) \supseteq \breve{\delta}$. It follows from the $(m, n)$-concavity of $\Gamma_{\breve{M}}$ that

$$
\gamma_{\breve{M}}\left(n \omega_{1}+m(1-n)^{\eta} \omega_{2}\right) \subseteq \gamma_{\breve{M}}\left(\omega_{1}\right) \cup \gamma_{\breve{M}}\left(\omega_{2}\right)
$$

and thus $\Gamma_{\breve{M}}^{\breve{\delta}}$ is a $(m, n)$-concave FSS in the $2^{\text {nd }}$ sense.

Conversely suppose that $\Gamma_{\breve{M}}^{\breve{s}}$ is a $(m, n)$-concave FSS in the $2^{\text {nd }}$ sense for every $n \in[0,1]$. For $\omega_{1}, \omega_{2} \in \breve{L}, \Gamma_{\breve{M}}^{\breve{\delta}}$ is $(m, n)$-concave FSS in the $2^{\text {nd }}$ sense for $\breve{\delta}=\gamma_{\breve{M}}\left(\omega_{1}\right) \cup \gamma_{\breve{M}}\left(\omega_{2}\right)$. Since $\gamma_{\breve{M}}\left(\omega_{1}\right) \subseteq \breve{\delta}$ and $\gamma_{\breve{M}}\left(\omega_{2}\right) \subseteq \breve{\delta}$, we have $\omega_{1} \in \Gamma_{\breve{M}}^{\breve{\delta}}$ and $\omega_{2} \in \Gamma_{\breve{M}}^{\breve{\delta}}$, hence $n \omega_{1}+$ $m(1-n)^{\eta} \omega_{2} \in \Gamma_{\breve{M}}^{\breve{\delta}}$. Therefore, $\gamma_{\breve{M}}\left(n \omega_{1}+m(1-n)^{\eta} \omega_{2}\right) \subseteq \breve{\delta}=\gamma_{\breve{M}}\left(\omega_{1}\right) \cup \gamma_{\breve{M}}\left(\omega_{2}\right)$, which indicates $\Gamma_{\breve{M}}$ is a $(m, n)$-concave FSS in the $2^{\text {nd }}$ sense on $\breve{L}$.

\section{CONCLUSION}

In this study, $(m, n)$-convexity and $(m, n)$-concavity are introduced under fuzzy soft environment, which is the extension of existing relative concepts. Moreover, some theoretic operations i.e. union, intersection and classical properties i.e. complement, $\breve{\delta}$-inclusion, are generalized. Some useful results are explored through different senses (known as 1st and 2 nd sense in literature) on $(m, n)$-soft convexity in this research work. Future work may include the extension of this concept to Interval-valued fuzzy soft sets, Intuitionistic fuzzy soft set, Pythagorean fuzzy soft set and many other fuzzy soft-like environments. This work will help the researchers to introduce certain types of convexity i.e Quasi, Pseudo, Graded, Triangular etc. under such environments.

\section{REFERENCES}

[1] M.I. Ali, F. Feng, X. Liu, W.K. Min, M. Sabir, On some new operations in soft set theory, Computers and Mathematics with Applications, 57 (2009)1547-1553.

[2] E. E. Ammar, Some Properties of Convex Fuzzy Sets and Convex Fuzzy Cones, Fuzzy Sets and Systems, 106, No.3 (1999)381386.

[3] K.V.Babitha, J.J.Sunil, Soft set relations and functions, Computers and mathematics with applications, 60(2010)1840-1849.

[4] K.V.Babitha, J.J.Sunil, Transitive closure and ordering in soft set,Computers and mathematics with applications, 61 (2011)2235-2239.

[5] N. Çă̆man, F. Çitak and H. Aktas, Soft int-group and its applications to group theory, Neural Computing and Applications, 21 (2012) 151-158.

[6] N. Çă̆man and S. Engino ğlu, Soft set theory and uni-int decision making, European Journal of Operational Research, 207 (2010) 848-855.

[7] N. Çă̆man, I. Deli, Means of FP-Soft Sets and Its Applications, Hacettepe Journal of Mathematics and Statistics, 41, No.5 (2012) 615625. 
[8] N. Çă̆man, I. Deli, Product of FP-Soft Sets and Its Applications, Hacettepe Journal of Mathematics and Statistics, 41, No.3 (2012), 365374.

[9] N. Çă̆man, S. Enginoğlu, F. C itak, Fuzzy Soft Set Theory and Its Applications, Iranian Journal of Fuzzy Systems, 8, No.1 (2011) 137147.

[10] B. B. Chaudhuri, Concave Fuzzy Set: A Concept Complementary to the Convex Fuzzy Set, Pattern Recognition Letters, 13, No.2 (1992) 103106.

[11] I. Deli, Convex and Concave Soft Sets and Some Properties, ResearchGate, (2013).

[12] I. Deli, Convex and Concave Sets Based on Soft Sets and Fuzzy Soft Sets, Journal of New Theory, 29 (2019) 101-110.

[13] P. Diamond, P. Kloeden, A. Vladimirov, Spikes, Broken Planes and the Approximation of Convex Fuzzy Sets, Fuzzy Sets and Systems, 99 (1998) 225232.

[14] D. Dubois, Fuzzy Weighted Averages and Fuzzy Convex Sums, Fuzzy Sets and Systems, 213 (2013)106108.

[15] X.Ge, S.Yang, Investigations on some operations of soft sets, World Academy of Science Engineering and Technology, 75 (2011)1113-1116.

[16] D. Hu, T. Jiang, and X. Yu, Construction of non-convex fuzzy sets and its application, Neurocomputing, 393 (2020) 175-186.

[17] Y. Jiang, Y. Tang, H. Liu, Z. Chen, Entropy on Intuitionistic Fuzzy Soft Sets and on Interval Valued Fuzzy Soft Sets, Information Sciences, 240 (2013) 95114.

[18] Koskela, Pekka, and V. Manojlović, Quasi-nearly subharmonic functions and quasiconformal mappings, Potential Analysis, 37, No.2 (2012), 187-196.

[19] P.K. Maji, R. Biswas, A.R. Roy, Fuzzy soft sets, Journal of Fuzzy Mathematics, 9, No.3 (2001) 589-602 .

[20] P.K. Maji, R. Biswas, A.R. Roy, Soft Set Theory, Computers and Mathematics with Applications, 45 (2003) 555-562.

[21] D. Molodtsov, Soft Set Theory - First Results, Computers and Mathematics with Applications, 37 (1999) 19-31.

[22] B. Pang, Bases and subbases in (L, M)-fuzzy convex spaces, Computational and Applied Mathematics, 39, No.2 (2020) 41.

[23] O. Pavlacka, Note on the Lack of Equality between Fuzzy Weighted Average and Fuzzy Convex Sum, Fuzzy Sets and Systems, 213 (2013) 102105.

[24] D.Pei, D.Miao, From soft set to information system, In:Proceedings of Granular computing, IEEE, 2 (2005) 616-621.

[25] D. N. Pinheiro, D. Aloise, and S. J. Blanchard, Convex fuzzy k-medoids clustering, Fuzzy Sets and Systems, (2020).

[26] A. U. Rahman, M. Saeed, and F. Smarandache, Convex and Concave Hypersoft Sets with Some Properties, Neutrosophic Sets and Systems, 38 (2020) 497-508.

[27] D. Sarkar, Concavoconvex Fuzzy Set, Fuzzy Sets and Systems, 79, No.2 (1996) 267269.

[28] M. Shabir, M. I. Ali, T. Shaheen, Another Approach to Soft Rough Sets, Knowledge-Based Systems, 40 (2013)7280.

[29] Todorčević, Vesna, Harmonic quasiconformal mappings and hyperbolic type metrics, Springer International Publishing, (2019).

[30] C. Wang, A. Qu, Entropy, Similarity Measure and Distance Measure of Vague Soft Sets and Their Relations, Information Sciences, 244 (2013) 92106.

[31] X. Wang, D. Ruan, E. E. Kerre, Mathematics of Fuzziness Basic Issues, Springer, (2009).

[32] Z. Y. Xiu, Q. H. Li, and B. Pang, Fuzzy convergence structures in the framework of L-convex spaces, Iranian Journal of Fuzzy Systems, 17, No.4 (2020) 139-150.

[33] X. Yang, Some Properties of Convex Fuzzy Sets, Fuzzy Sets and Systems, 72, No.1 (1995) 129132.

[34] L. A. Zadeh, Fuzzy sets, Inf Control, 8 (1965) 338-353.

[35] S. Y. Zhang, S. G. Li, and H. L. Yang, Three-way convex systems and three-way fuzzy convex systems, Information Sciences, 510 (2020) 89-98. 